\title{
Contra el Androcentrismo Curricular y por una Educación Sexuada Justa
}

\section{Against Curriculum Androcentrism and for a Fair Sex Education}

\author{
Graciela Morgade * \\ Universidad de Buenos Aires
}

\begin{abstract}
Desde hace décadas las diferentes expresiones del feminismo vienen denunciando que los estudios críticos de la educación no son lo suficientemente críticos si no incorporan los análisis desde la perspectiva de género. El artículo presenta avances de una investigación en curso dirigida a producir innovaciones educativas desde la perspectiva de géneros y derechos humanos. Se trata de un dispositivo de investigación acción participativa, desarrollado en el marco de políticas de desarrollo de una Educación Sexual Integral de Argentina, que encuentran un límite resistente en los saberes de referencia académica del programa de estudios escolar. Se trabajó con profesoras de Formación Ética y Ciudadana, Lengua y Literatura y Biología y Educación para la Salud. Los primeros resultados señalan desiguales puntos de desarrollo en las diferentes disciplinas con una resistencia particularmente fuerte en las Ciencias Naturales. Las tensiones innovadoras se fundamentan generalmente en la crítica de los supuestos disciplinares desde la epistemología feminista, subrayando la dimensión situada, histórica y cultural de las ciencias. La lucha dentro de la academia y dentro del sistema educativo se nutre de los debates sociales que los movimientos colocan; pero esos debates sociales necesitan su incorporación epistemológica estructural en los saberes y en el sistema educativo para tener una sustentabilidad que transcienda los límites temporales y personales para integrarse en el proyecto de una educación sexuada justa.
\end{abstract}

Descriptores: Sexo, Plan de estudios, Educación sexual, Movimiento de liberación femenina, Justicia

For decades, the different expressions of feminism have been denouncing that critical studies of education are not sufficiently critical if they do not incorporate analyzes from the gender perspective. The article presents advances of an ongoing research aimed at producing educational innovations from the perspective of genres and human rights. It is a participatory action research device, developed within the framework of policies of development of an Integral Sexual Education of Argentina, that find a limit resistant in the academic reference knowledge of the school curriculum. We worked with teachers of Ethics and Citizenship, Language and Literature and Biology and Health Education. The first results indicate uneven developmental points in the different disciplines with a particularly strong resistance in the Natural Sciences. Innovative tensions are generally based on the critique of disciplinary assumptions from feminist epistemology, emphasizing the situated, historical and cultural dimension of the sciences. The struggle within the academy and within the educational system is nourished by the social debates that the movements place; but these social debates need their structural epistemological incorporation in the knowledge and in the educational system to have a sustainability that transcends the temporal and personal limits to be integrated in the project of a fair sexual education.

Keywords: Sex, Curriculum, Sex education, Womens liberation movement, Justice.

Apoyado por Programa UBACyT de la Universidad de Buenos Aires.

*Contacto: gmorgade@filo.uba.ar

ISSN: 2254-3139

www.rinace.net/riejs/

revistas.uam.es/riejs
Recibido: $\quad 17$ de mayo 2017

$1^{\text {a }}$ Evaluación: 4 de julio 2017

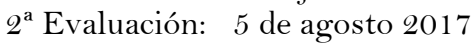

Aceptado: $\quad 3$ de septiembre 2017 


\section{Introducción}

Desde hace décadas ya, las diferentes expresiones del feminismo vienen denunciando, sistemáticamente, que los estudios críticos de la educación no son lo suficientemente críticos si no incorporan los análisis desde la perspectiva de género. El campo de investigación y las herramientas de interpretación se ampliaron desde entonces. Empleando categorías como la de reproducción o de la resistencia a la imposición de formas culturales de las clases dominantes, la conceptualización de la escuela como aparato ideológico de estado, o la recuperación de la noción gramsciana de hegemonía, los resultados de los estudios fueron consistentes en identificar: la persistencia de significaciones patriarcales tanto en el currículum formal prescripto por la administración educativa, como en el llamado currículum oculto, es decir, las expectativas de rendimiento y comportamiento hacia mujeres y varones, y en las omisiones sistemáticas de temas relevantes para la vida personal o profesional de las mujeres, el llamado currículum omitido, aquello de lo que no se habla o se habla a través del silencio: la sexualidad, el deseo, la violencia de género o la precarización laboral femenina (Morgade, 2011).

El artículo presenta avances de una investigación en curso dirigida a producir innovaciones educativas desde la perspectiva de géneros y derechos humanos. Se trata de un dispositivo de investigación acción participativa, desarrollado en el marco de políticas de desarrollo de una Educación Sexual Integral de Argentina, que encuentran un límite resistente en los saberes de referencia académica del programa de estudios escolar. Se trabajó con profesoras de Formación Ética y Ciudadana, Lengua y Literatura y Biología y Educación para la Salud. Los primeros resultados señalan desiguales puntos de desarrollo en las diferentes disciplinas con una resistencia particularmente fuerte en las Ciencias Naturales. Las tensiones innovadoras se fundamentan generalmente en la crítica de los supuestos disciplinares desde la epistemología feminista, subrayando la dimensión situada, histórica y cultural de las ciencias.

\section{Antecedentes}

\section{1. Educación, género y sexualidades en la escuela secundaria}

Las investigaciones en el nivel secundario de la educación evidenciaron la tendencia a la reproducción, por presencia o ausencia, de la significaciones hegemónicas en el sistema sexo-género en la mayoría de las áreas académicas: la historia centrada en la celebración de los héroes militares o políticos (López Navajas, 2014), el canon de la literatura que solo admite grandes obras consagradas y escritas por varones (Lasa Álvarez, 2016), el lenguaje sexista enseñado en la escuela sin mediación de crítica (López Valero, Madrid Izquierdo y Encabo Fernández, 1998), contenidos para chicas y contenidos para chicos en la Educación Física (Scharagrodsky, 2006), las omisiones y los prejuicios en el caso de las ciencias exactas y naturales (Moss-Racusin et al., 2012). Los nudos de sentido que fundamentan esos sesgos sexistas patriarcales también fueron ampliamente analizados: por una parte, la evocación a la naturaleza como destino para los cuerpos sexuados; de manera complementaria, el silencio respecto del sistema sexogénero de construcción, legitimación y ejercicio del poder. Estas mismas indagaciones en el campo de los estudios de Géneros y Educación mostraron los puntos de fuga, las resistencias y, sobre 
todo, el enorme diferencial que la posibilidad de incorporarse, permanecer y finalizar estudios formales representa para las vidas de las mujeres.

Paralelamente, en un campo de extraordinaria vitalidad y sin dejar de reconocer su potencia, se desarrolló el cuestionamiento del binarismo de la categoría género que dejaba sin criticar el supuesto de que existen dos cuerpos (de varones y mujeres) a los que les corresponde dos géneros (masculino y femenino) y una direccionalidad correcta, normal del deseo por el cuerpo/género opuesto (Butler, 2002). Se habilitó entonces la construcción de nuevos objetos de investigación que comenzaron a evidenciar que en las prácticas escolares además de predominar la invisibilización o estereotipación de lo femenino y lo masculino no hegemónico, predomina la tendencia a condenar por abyectas a todas las formas identitarias que no se adecuen a la normal coincidencia entre dotación biofísica del sexo (externa), deseo y formas culturales de vida.

Se incorpora entonces con fuerza el estudio de la cuestión de la construcción social del cuerpo sexuado y las sexualidades y sus articulaciones y desarticulaciones con el sistema sexo-género. Trabajos pioneros como la obra de Debbie Epstein y Richard Johnson (1998) en Gran Bretaña, Deborah Britzman (1999) y Michelle Fine (1999) en Canadá, Guacira Lopes Louro (1999 y 2004) en Brasil. En la Argentina, orientadas hacia el análisis de las experiencias de docentes heterosexuales y lesbianas (Alonso, Herczeg y Zurbriggen, 2008) han abordado la relación heterosexualidad / homosexualidad en las prácticas escolares y en las representaciones de mujeres docentes. Jesica Báez (2013), Luciana Lavigne (2016), Juan Péchin (2013, 2014) en Argentina configuran una nueva generación de jóvenes investigador@s en el campo.

Estos trabajos muestran el sesgo racionalista de la educación formal en Argentina en la que los cuerpos (de tod@s) resultan uno de sus silencios sistemáticos. Las investigaciones hicieron posible encontrar al cuerpo en crudos estereotipos de género, en el aula y en el patio y, sobre todo, la educación física (el cuerpo militarizado, (Scharagrodsky, 2006, 2008) y la educación artística (el cuerpo sentimentalizado), especialmente en la recientemente incorporada al currículum expresión corporal (López Cao, 2002). El valor predominante del cuerpo de la educación física es el del alto rendimiento, la alta competencia y la fuerza de los equipos de primera o del atleta en la antigua Grecia; las mujeres y muchos varones quedan afuera. Si se trata de expresividad en cambio, se habla de comunicación, de mundo interior y, de alguna manera, se alude a las significaciones de lo femenino; aquí son los machos los discriminados, y las identidades trans son totalmente negadas. Un compromiso muy elevado de un varón en la expresión corporal puede llegar a provocar burlas y diversas formas de violencia vinculadas con su orientación sexual (Acosta, Cuellar y Martínez. 2013). Así se refuerza el ideal identificatorio; para los varones: transgresores, valientes, arriesgados, poco sensibles y, menos comunicativos o expresivos; y siempre heterosexuales; para las mujeres, expresividad, delicadeza, sensibilidad...siempre heterosexuales también.

Ahora bien, uno de los ejes que organizan los resultados de todos estos antecedentes es la hipótesis de que la escuela, siempre, por acción u omisión, desarrolla contenidos y mensajes relativos de los cuerpos sexuados. Es decir, que la educación siempre es sexual. El proyecto político sería que se reconozca como tal, es decir una educación sexuada, y que la crítica señale si esa educación sexuada se dirige hacia un proyecto de justicia o hacia un proyecto patriarcal, heteronormativo y transfóbico (Connell, 1997; Morgade, 2011). 
Volviendo a colocar a la cuestión de la fuente académica del currículum escolar, los saberes de referencia (Perrenoud, 2001) como uno de los factores más poderosos que tiene la escuela y, por lo tanto, uno de los limites más resistentes para cualquier proyecto de educación sexuada justa, hemos encarado desde 2014 una investigación que tiende a producir innovaciones pedagógicas y curriculares en la práctica escolar cotidiana, trabajando en conjunto con profesoras de algunas áreas que se enseñan en las escuelas en el nivel secundario, cuyos avances sistematizamos en el presente artículo.

\subsection{En torno a Ley de Educación Sexual Integral (ESI) en Argentina}

En 2006 se votó en Argentina una Ley de Educación Sexual Integral. Su reglamentación se produjo en 2008 mediante la votación de lineamientos curriculares que representan decisiones explícitas respecto de los modos en que el proyecto debe ser llevado a cabo. La recurrente discusión en relación a la inclusión curricular de la perspectiva de género y las sexualidades entre su abordaje como eje transversal, como instancia específica o como temas a desplegar en algunas unidades curriculares está respondida en los lineamientos curriculares estableciendo que debe abordarse como un enfoque general y temáticas explícitas en todas las materias y en espacios específicos desde el nivel secundario y en la formación docente.

Si bien la sanción de la ley constituyó un extraordinario avance en el marco de las luchas de los movimientos feministas y sociosexuales en general, esta reglamentación tuvo desde el inicio al menos tres dimensiones para atender como limitaciones posibles.

Las materias escolares en la tradición enciclopédica y racionalista del profesorado tienen un correlato en una fuerte fragmentación del trabajo, sobre todo en la escuela secundaria, obstaculizando el trabajo en equipo inter o multidisciplinario (Lenoir, 2013). El profesorado entonces se ve limitado a agregar temas específicos, con importantes dificultades de integración interdisciplinaria e institucional, lo cual implica con frecuencia fuertes reducciones sustantivas vinculadas con la caja horaria escolar y los tiempos de clase. Además, si se toma la transversalización de género como principio que trabaja sobre las distintas dimensiones que puedan tener impactos negativos en términos de las relaciones de género, esta implicaría ir más allá del currículo explícito para abordar a la institución en su conjunto. En esta línea una organización que propone la transversalización revisaría constantemente aspectos tales como: los objetivos de la institución y particularmente de los espacios de atención en salud sexual y reproductiva, las reglas de funcionamiento, los modos de comunicación y participación, la distribución de los espacios físicos, la asignación de recursos.

Por otra parte, es sabido que no es suficiente con la crítica de los procesos de construcción y selección de los saberes a trabajar en las aulas. Se trata también de las formas que toma el contenido: el lugar de los saberes y las experiencias. Edwards (1995) sostiene que el contenido no es independiente de la forma en la cual es presentado. La forma tiene significados que se agregan al contenido transmitido, produciéndose una síntesis, transformando los contenidos y produciendo uno nuevo. El contenido se transforma en la forma. Además, y ya desde el movimiento de mujeres a través de la pedagogía de la diferencia y su versión de la pedagogía feminista (Maher y Thompson, 2001), y también desde los movimientos de educación popular latinoamericanos, se iluminó otra dimensión de la cuestión: problema de la educación reside fundamentalmente en el proceso de enseñanza y aprendizaje. Su foco está colocado en el nivel del aula (Piussi, 1989) y planteó muy tempranamente lo insuficiente de un concepto 
de igualdad ligado a un modelo masculino y la necesidad de atravesar ese concepto con la categoría de la diferencia, para dejar emerger una nueva definición de la justicia que posteriormente Nancy Fraser (1995) conceptualizaría como de doble sentido: redistribución y reconocimiento.

La pedagogía de la diferencia sostiene entonces que la premisa universalista del pensamiento moderno, el derecho a educarse/instruirse, puede dejar de ser una ficción si se construye un orden educativo diferenciado. Algunas teóricas de los movimientos sociosexuales también vienen trazando algunos caminos para seguir estas pistas: Deborah Britzman (1999) en particular, que identifica tres versiones de la educación sexual: la normal, normalizadora de los cuerpos y el deseo; la crítica, que cuestiona a las relaciones de poder y reivindica los derechos sexuales y reproductivos y la que denomina la aun no tolerada, que apunta al cuidado de sí como práctica de la libertad. Esta pedagogía no resulta tolerada no solamente por su carácter radicalizado en términos de géneros, sino porque la estructura escolar vigente en la que la evaluación es sinónimo de control de lecturas y no tiene otras herramientas para desplegarse.

Por último, la transversalización, principal objeto de nuestra línea de investigación actual, apela también a las formas en que cada disciplina en particular se ha hecho cargo de la crítica desde la perspectiva de género, sin que se haya producido de manera equivalente en cada campo la crítica epistemológica al androcentrismo de las ciencias. Reiteramos, estas limitaciones no invalidan el proyecto de la Educación Sexual Integral. Sólo lo ubican como una política educativa de largo aliento, que no se resuelve en los pocos años que dura una administración, sino que se trata de una política de estado, amenazada por la discontinuidad que el gobierno asumido en 2015 imprimió al conjunto de iniciativas nacionales.

\section{3. EI terreno donde la ESI siembra}

Ahora bien, cualquier proyecto educativo se realiza en un territorio, la escuela, con historias, tradiciones y experiencias. Como casi ningún otro proyecto, la ley de Educación Sexual Integral se asienta en un terreno nada neutral. No se trata solamente de los sentidos hegemónicos acerca de los cuerpos sexuados (aludidos en la presentación). Existen también antecedentes vinculados explícitamente con la tematización de la sexualidad y el cuerpo humano en las aulas escolares.

En la Argentina, una de las tradiciones de mayor presencia en el tratamiento escolar de las temáticas vinculadas con las sexualidades, es el modelo denominado biologicista. Desde esta perspectiva, se considera que se abordan las cuestiones de la sexualidad si se estudia la anatomía y la fisiología de la reproducción desgajada de las emociones o de las relaciones sociales que dan sentido al uso del cuerpo biológico. La negación de que en toda forma de clasificación de lo natural están subyaciendo categorías sociales de significación, lleva también a clasificar entre normales y, por ende, anormales a las configuraciones corporales intersex y abyectas (Butler, 2004) a todas las formas de elección de objeto sexual no funcionales a la reproducción de la especie (Colectivo LGBT).

Este abordaje suele complementarse con una perspectiva médica que organiza el estudio del cuerpo biológico bajo un enfoque centrado en la prevención. En el discurso escolar se apela a la prevención en tres nudos de sentido diferentes (Morgade, 2011): 
- Prevención como cuestión del cuerpo humano. Prevenir un cuerpo que se puede embarazar, enfermar, hacerse adicto, alcoholizarse, suicidarse. Se concibe a un cuerpo desenfrenado que es necesario contener. Considerado natural y por lo tanto pre-social y con ello anterior a las relaciones de poder/saber, la prevención se afinca en dominarlo a fin de lograr humanidad en él. La biologización escolar de las sexualidades funciona entonces como dispositivo de disciplinamiento social de los cuerpos, abonando y reforzando la medicalización.

- Prevención como cuestión de expert@s. Se recurre a 1@s especialistas, a aquell@s que son poseedores/as del recurso de experticia que legitima su actuar de forma técnica (y despolitizada). Desde la Medicina o la Psicología, cuando se trata de especialistas extraescolares; y, en general, 1@s profesores/as de Biología dentro de la escuela.

- Prevención como cuestión de mujeres y de heterosexuales. Desde esta perspectiva las principales destinatarias son las alumnas, pues ellas se embarazan, pueden ser abusadas y violadas, deben visitar al ginecólogo.

Así, cuando se habla de la prevención, se mira y se piensa la sexualidad desde una visión patologizante: la sexualidad como situación de riesgo, o de enfermedad.

Este paradigma biomédico es hegemónico; por ello, la materia por excelencia para tramitar la educación sexual ha sido tradicionalmente Biología y Educación para la Salud. Según Elsa Meinardi (2008), el currículum de la Formación Docente no ha incluido hasta el presente contenidos dirigidos a encarar la enseñanza de las temáticas de salud sexual desde una perspectiva integral y no medicalizante. Evidentemente, no se trata de la ausencia o el silencio de los discursos sobre las sexualidades en la formación docente inicial, sino que el enfoque que enmarca su abordaje y enseñanza en el ámbito pedagógico y educativo obtura la superación del reduccionismo biomédico que supone agotar la sexualidad en la genitalidad.

Es posible identificar también otra tradición, denominada moralizante o disciplinadora, fuertemente inspirada en principios religiosos y, en general, predominantemente de orientación católica. En esta tradición, presente no solamente en las instituciones de educación confesional (Wainerman, Di Virgilio, Chami, 2008), la mirada sobre las sexualidades también las coloca en una dimensión de problema: la amenaza del descontrol deberá atenderse con la castidad, la amenaza de los embarazos no planificados, con la abstinencia. Esta tradición sigue sosteniendo que existen dos cuerpos normales y que el matrimonio tiene una sola forma: la unión entre un varón y una mujer.

Lo interesante y desafiante es que estas tradiciones no se corresponden término a término con la modalidad de gestión de los establecimientos escolares: tanto en escuelas de gestión pública estatal como en escuelas confesionales religiosas se encuentra una combinación de estas miradas hegemónicas.

El terreno donde la ESI siembra entonces, como en cualquier otro caso de política educativa, pero con más intensidad aún, en los saberes y experiencias previas del sector docente. 


\section{Hacia la construcción de lo nuevo: una investigación acción participativa}

El proyecto que fundamenta los resultados que se presentan a continuación adopta como dispositivo metodológico a la tradición de la investigación acción participativa, enmarcada en los Estudios Culturales y de Género.

La investigación pretende producir conocimientos de situaciones y procesos en profundidad desde una indagación participativa donde el proceso sea mediado por un colectivo formado entre este equipo de investigación y docentes-investigadores/as. Se procura entonces, una participación real de la población involucrada (Achilli, 2000; Batallan y García, 1988; Sirvent, 1993).

Se fundamenta, a su vez, en la tradición originada en la grounded theory y los desarrollos del interpretativismo en la etnografía. En este sentido, se apunta a desplegar teórica y empíricamente el problema de investigación, tendiendo reconstruir la perspectiva de los actores sociales procurando ampliar la interpretación en tensión con los procesos histórico-culturales más amplios.

El diseño consiste básicamente en un estudio de casos, desde la programación hasta el desarrollo de propuestas didácticas. Los casos seleccionados se encuentran en la Facultad de Filosofía y Letras y en la Facultad de Ciencias Exactas y Naturales de la Universidad de Buenos Aires y en la Facultad de Humanidades y Ciencias de la Educación de la Universidad de La Plata. Se trata de tres cátedras (una en cada Facultad) integradas en todos los casos por una o dos profesoras a cargo y un equipo de entre cuatro y seis docentes auxiliares.

La investigación se propone conocer la significación explícita que dan los sujetos a la formación y las prácticas docentes que derivan de la misma, poniendo de manifiesto su mutua relación. Desde esta orientación, la reflexividad que los agentes tengan de los condicionantes de su vida social -y que los convierte en sujetos-, es una condición necesaria, aunque no suficiente, para la transformación social. En el marco de la conceptualización, anteriormente explicitada, el enfoque antropológico hará su particular aporte entendiendo que la investigación debe mostrar cómo se establecen los nexos que permiten reconstruir la lógica informal de la vida cotidiana y las estructuras históricamente conformadas. El trabajo de participación y distanciamiento es paralelo entonces a la exploración de la propia experiencia, que entendemos también representa una fuente de saberes válidos.

La investigación aspira a integrar en el análisis el plano de las políticas y las determinaciones normativas, que constituyen el marco de las prácticas de los sujetos, el plano de las significaciones culturales que en la vida cotidiana social y escolar tienden a preservar la hegemonía de una definición de género en detrimento de otras y el plano de los modos en que los sujetos responden en línea o en oposición al disciplinamiento sobre sus prácticas y, en consecuencia, sobre sus cuerpos. En las diferentes estrategias metodológicas, se analizan los datos en dos momentos. Un primer momento implica la reconstrucción de cada caso según las categorías de organización de sentido propias de los actores sociales en tensión con los interrogantes planteados por la investigación. En un segundo momento, se elaboran el análisis e interpretación propiamente, presentados como resultados: el análisis retoma los interrogantes y los conceptos orientadores de la 
investigación de modo de recuperar o reconstruir categorías conceptuales de un orden de mayor generalidad (no estadística sino teórica) que organizan la interpretación.

\section{Algunos resultados de nuestra investigación: el duro núcleo de las disciplinas}

\section{Vacancias en la formación del profesorado y los equipos técnicos centrales}

Un primer hallazgo de la investigación es que la crítica epistemológica de los saberes de referencia de los contenidos escolares se ha venido desarrollando en la academia en un volumen y velocidad que no se acompasó con la transformación curricular en el sistema educativo ni en la formación del profesorado.

Por una parte, anticipamos, porque el proceso de validación en los espacios universitarios es un proceso inacabado: los estudios de géneros son marginales aún en muchos planes de estudio en los ámbitos de formación de docentes. Esto implica que el profesorado llega a trabajar a las escuelas sin una formación previa que permita anclar la propuesta que la Ley de Educación Integral implica. La recontextualización de contenidos que se realiza en el establecimiento escolar y en cada aula, se articula con los escasos saberes previos de la formación docente y también con las ideas, valores y creencias asentados como sentido común docente en tanto sujetos sociales, en que inciden de manera muy diversa tanto los proyectos de formación continua ofrecidos al profesorado como la experiencia de la militancia y la lucha de los movimientos feministas y sociosexuales en general que tensan a la opinión pública (Morgade y Fainsod, 2015).

Por otra parte, porque los diferentes espacios de recontextualización de esos saberes de referencia también tienen lógicas y visiones que sospechan e impugnan con frecuencia los desarrollos de la academia: los equipos técnicos centrales y locales del sistema educativo, que definen los núcleos, parámetros o lineamientos, también operan con frecuencia descartando o reduciendo los aportes de las producciones de los feminismos y los movimientos sociosexuales en general.

Desigual desarrollo en las diferentes disciplinas, resistencia en las Ciencias Naturales. Los importantes desarrollos teóricos acerca de la construcción social del cuerpo sexuado que han tenido lugar en las Ciencias Sociales (sobre todo, la Historia) son los de mayor presencia y potencia en la implementación de la Ley. Sin embargo, suelen presentarse como una incorporación compensatoria o como problema; en el terreno afín, la Formación Ética y Ciudadana (campo de la Filosofía y Derecho), la incorporación suele reducirse al carácter de tema especial. El caso de la Lengua y la Literatura presenta una situación intermedia, ya que se trata de un área en la cual los materiales de lectura recomendados suelen ser significativos en términos subjetivos y sociales. La asignatura aparece como clave para el abordaje de temáticas relevantes para la justicia de género, pero más como una mediación didáctica que a partir de una mirada epistemológica autocrítica (Baez, Melo y Malizia, 2017).

Las áreas más resistentes parecen ser las Ciencias Naturales (campo de la Biología y Educación para la Salud), instancias curriculares que se han erigido como los espacios de la Educación Sexual por antonomasia, con su carga autoadscripta de valoración científica y cientificista. La mirada hegemónica sostiene que el sexo de una persona queda 
determinado a partir de los cromosomas sexuales, se habla de caracteres sexuales primarios determinados por la posesión de un determinado sistema genital y caracteres sexuales secundarios según la presencia durante el desarrollo de hormonas sexuales masculinas y femeninas, apelando a una existencia natural de los sexos. Escasa o nula es la crítica de la clasificación entre cuerpos y sexualidades naturales o normales y antinaturales o anormales, en particular, a todas las formas de elección de objeto sexual no funcionales a la reproducción biológica de la especie y/o a toda configuración corporal que no se encauce dentro del binarismo macho-hembra (Grotz, 2016).

\section{Nudos transformadores en la formación ética y ciudadana}

Los silencios en los diferentes documentos de base de los que disponen profesores y profesoras evidencian que las producciones del movimiento social de mujeres y los movimientos sociosexuales no terminan de ingresar plenamente para dar direccionalidad a la enseñanza. Las oportunidades de formación complementaria del profesorado hasta el momento dejan bajo el interés o la responsabilidad, y aún la opción, de cada docente completar el sentido y concretar la integralidad en la implementación de la Ley de Educación Sexual.

En términos pedagógicos, la interpelación desde la ESI se articula con debates en el propio territorio de la enseñanza de esta área.

Si la enseñanza se reduce a un conocimiento enciclopédico de los derechos, éstos devienen instrumentos para defenderse de los ataques y reclamar al estado que los garantice. Se trata de un nivel sin duda importante y valioso, con la limitación de que, si se estimula una relación externa con ellos, los derechos devienen abstractos, sin cuerpos ni deseos que los encarnan. Así, no terminan de resultar un medio para el proceso subjetivante de sí mismo/a y de1@s otr@s de concebirse y concebir al otr@como sujeto de derecho y sujeto de deseo, objetivo que la Ley de ESI tiene en sus fundamentos y en la historia de las luchas que le dieron sustento.

Si la enseñanza apunta a la dimensión deliberativa de los contextos que dieron lugar a la emergencia de los derechos humanos y su ampliación progresiva, el dispositivo de la clase puede transformarse también en un escenario de ejercicio de la propia voz, situada y corporizada, en la construcción, desde una posición de autonomía, de un conocimiento que incluya a las leyes en tanto construcción histórica. Las experiencias desarrolladas apuntaron entonces a corporizar el campo de los derechos, problematizando la cuestión socio-histórica, económica y cultural de su devenir a lo largo de la historia,

\section{Nudos transformadores en la enseñanza de lengua y literatura}

Tal como hemos anticipado, la asignatura resulta un lugar fértil de anclaje específico para el trabajo de la propuesta de la Ley de ESI. Así, la producción curricular iniciada desde la sanción de leyes de Educación Sexual ha sido creciente. No obstante, parecería que las producciones realizadas en ese marco se mantienen en los márgenes y no logran atravesar la interesante direccionalidad que el enfoque de prácticas del lenguaje ha dado hacia la experiencia de $1 @$ s estudiantes. Desde esta perspectiva, se entiende que la participación en la comunidad implica no sólo la posibilidad de formular opiniones, sino que también en el mismo acto se construye la identidad social y que la escuela resulta entonces un escenario privilegiado para dar lugar a la voz y su construcción. Partiendo de que no se nace con voz, sino que se llega a tenerla, la institución escolar resulta central (aun cuando no sea la única) en tanto contribuye a dotar a las personas de 
identidad política. Por otra parte, en la tradición escolar moderna la voz docente se sedimentó como voz autoritaria, la que es expositiva explicativa, la que sabe todo y coloca en1@s otr@s un saber nada, donde el conocimiento es racional, desgajado de las emociones e intuiciones. Desde una pedagogía feminista, la primera cuestión es levantar la voz, y que esto implica el desarrollo de la identidad de 1@s estudiantes como individuosgenerizad@s.

Las experiencias concretas nos han permitido modelizar (Baez, Melo y Malizia, 2017) algunos aspectos para hoy abrir al debate y dar lugar a la diversidad.

Incorporar el género, incorporando textos específicos: En este formato, la incorporación de contenidos de Educación Sexual Integral se encuentra sostenida desde la selección de textos que explícitamente desarrollen temáticas vinculadas. Consecuentemente, el objetivo desde la docente es incorporar un tema que le preocupa por medio de una obra literaria. El texto se presenta entonces como el organizador/contenedor de los contenidos a desplegar en el aula, pudiéndose establecer distintas estrategias para abrir dicho abanico.

Incorporar el género, construyendo una mirada crítica: Otra forma de incorporar la Educación Sexual Integral se delinea al delimitar como objetivo de tal acción, la construcción de una mirada crítica en 1@s estudiantes que les permita desnaturalizar las posiciones que ocupan en tanto sujetos sexuados en el campo social. Bajo esta dirección, se seleccionan una serie de contenidos que por medio de diversos materiales y actividades son explorados. Esta construcción por otra parte no solo incluye la posibilidad analítica racional del afuera sino también la inclusión de la propia experiencia como objeto de reflexión. Se trata de una mirada situada y en este sentido, no solo aspira a los contenidos racionales, sino que avanza sobre aspectos sentimentales y vinculares.

Incorporar el género, creando una escritura propia: Un último formato que pudimos vislumbrar se vincula al propósito de explorar las posibilidades de expresión en tanto sujet@s sexuados. Corriéndose del énfasis puesto en el análisis de género de las obras literarias, la propuesta se sitúa en poder hacer uso de las obras literarias como disparador de la propia escritura dando cuenta que la misma es una construcción de un narrador, o narradora, que escribe desde una inscripción sexuada. Diferenciando entre autor/a y narrador/a, y mediando entre uno y otras diversas herramientas literarias, la escritura y su desarrollo es conceptualizado desde la inscripción creativa de los cuerpos posibles. Dando lugar, también al cuestionamiento de los parámetros que definen tal posibilidad.

Nudos transformadores en la enseñanza de la biología y la educación para la salud

En el caso de la Biología, se ha pretendido llevar a la práctica la incorporación de nuevas miradas y nuevos métodos de enseñanza, de forma que se habilite el trabajo en profundidad de problemáticas vinculadas a las desigualdades sociales en general y a las de género en particular.

Es posible enseñar de manera no patriarcal ni heteronormativa los temas clásicos del área, tales como el proceso de hominización, la evolución de la especie, la genética y, sobre todo, la cuestión de las hormonas y las determinaciones de los sexos (González y Busca, 2017). Siguiendo a Fausto Sterling (2006) y otras teóricas del campo, entendemos que cuanto más buscamos una base física simple para definir el sexo, más claro resulta que sexo no es una categoría puramente física o biológica, que las señales y funciones 
corporales que definimos como masculinas y femeninas están ya presentes en nuestras concepciones del género.

Dentro de un campo afín al de Biología, en Educación para la Salud, se apunta a habilitar conversaciones en el aula a partir de una pedagogía cuyos rasgos distintivos sean asumir explícitamente la parcialidad de las perspectivas, los límites del saber, el no saber; habilitar a lo que se quiere saber, las respuestas que aún no se encuentran y las que se suponen singulares; tentar a 1@s estudiant@s a explorarlas y explorarse. Así, una premisa de trabajo es la crítica de los conceptos hegemónicos de salud y de enfermedad entendiendo la articulación indisociable entre la dimensión biofísica, la dimensión emocional subjetiva y su carácter social y político. Los desarrollos novedosos implicaron la construcción de contenidos y enfoques dirigidos a garantizar el efectivo ejercicio de los derechos, problematizando desde esa mirada en abordaje de las infecciones de transmisión genital, el acoso sexual, el abuso y la violencia sexual, el maltrato, la explotación sexual y trata, el aborto, que en Argentina está condenado y constituye una de las prácticas de mayor desigualación entre las mujeres que abortan, algunas en condiciones de asepsia y seguridad totalmente protegidas y otras poniendo en riesgo sus vidas, o muriendo (Fainsod y Busca, 2016). Evidentemente se hace necesaria una mirada multidisciplinara que incluya a las ciencias humanas y sociales en estos abordajes.

\section{Primeras conclusiones de una investigación en curso}

En el marco de la Ley de Educación Sexual Integral reglamentada en 2008 y votada en 2006, las experiencias curriculares que abordan contenidos innovadores en el campo de las relaciones de género y las sexualidades interpelan al orden escolar. Sin embargo, las formas institucionales en que se organiza la escuela argentina dificultan pensar al carácter integral de la sexualidad y a las formas pedagógicas inclusivas de los diversos cuerpos sexuados presentes en la escuela. Por otra parte, el desigual desarrollo de la crítica epistemológica intradisciplinaria implica una incorporación escolar de la ESI en forma de temas puntuales, que se yuxtaponen al núcleo duro de los contenidos de cada disciplina, restando el potencial aporte de la epistemología feminista a la transformación del conocimiento y enseñanza escolares.

Es evidente que las diferentes áreas del curriculum escolar tienen aportes específicos para el proyecto de la Educación Sexual Integral con enfoque de géneros y derechos humanos. Sin embargo, la investigación va mostrando cuestiones comunes a todas las asignaturas: la crítica epistemológica interpela tanto a los objetos y enfoques teóricos con que las ciencias investigan (contenidos constitutivos de los saberes de referencia) como al recorte que las diferentes oficinas de la administración educativa realizan de esos desarrollos para conforman el curriculum escolar.

La sustentabilidad de estas políticas parece estar aún asentada en la militancia. Es para reflexionar de qué modo un proyecto que nace inspirado en las demandas del movimiento social de mujeres y los movimientos sociosexuales, plasmadas por ejemplo en sucesivos documentos producidos en conferencias nacionales e internacionales es reconocido como movimiento social, cómo acumula poder y predicamento en la sociedad. Una cuestión para pensar es que una política pública que aspire a ser efectiva no puede ser feminista. Sí puede y debe tener una agenda o una perspectiva feminista o trans de 
mirar el mundo... pero no requerir ser militante para ser comprendida e implementada. En otras palabras, que el límite de la política sea sus propios dogmas...

Es evidente también que los modos microfísicos en que una política se encarna trascienden fuertemente a los grupos promotores e inclusive a la duración de las políticas llevadas adelante por los gobiernos. Las iniciativas vuelven en manifestaciones nuevas y con nuevo vigor. En nuestro país, las leyes de matrimonio igualitario y de identidad de género hicieron mucho más por la ESI que muchos cursos de capacitación o muchos materiales específicos.

Así, tampoco esta política parte solamente de las controversias entre actores y producciones institucionales, de las fuentes académicas del currículum y sus sucesivas recontextualizaciones ni inclusive solo de los procesos cotidianos de las aulas en las que se enseña y se aprende. En este caso al menos, también incide la opinión pública (o, con mayor precisión, publicada) y las formas en que se visibilizan y tematizan los conflictos sociales vinculados con el sistema sexogénero. En otras palabras, la lucha dentro de la academia y dentro del sistema educativo se nutre, se inspira, se alimenta, de los debates sociales que los movimientos colocan; pero esos debates sociales necesitan su incorporación epistemológica estructural en los saberes y en el sistema educativo para tener una sustentabilidad que transcienda los límites temporales y personales para integrarse plenamente en el proyecto de una educación sexuada justa.

\section{Referencias}

Achilli, E. (2000). Investigación y formación docente. Rosario: Laborde.

Alonso, G., Herczeg, G. y Zurbriggen, R. (2008). Talleres de educación sexual. Efectos del discurso heteronormativo. En G. Morgade y G. Alonso (Comps.), Cuerpos y sexualidades en la escuela. De la normalidad a la disidencia (pp. 105-136). Buenos Aires: Paidós.

Acosta Posada, M., Cuellar Wills, L. y Martínez Orozco. J. (2013). Colombia: El bullying por homofobia debe salir del clóset. Bogotá: Fundación Sentido.

Baez, J. (2013) La experiencia educativa trans. Los modos de vivir el cuerpo sexuado de l@s jóvenes en la escuela secundaria (Tesis doctoral). Universidad de Buenos Aires.

Baez, J., Melo, M. y Malizia, A. (2017). Generizando la lengua y la literatura desde la codidianeidad del aula. Rosario: Homo Sapiens.

Batallan, G. y Garcia, J. (1988). Problema de la investigacion participante y la transformación de la escuela. Rosario: Universidad Nacional de Rosario.

Britzman, D. (1999). Curiosidade, sexualidade e curriculo. En G. Lopes Louro (Ed.), O corpo educado. Pedagogias da sexualidade (pp. 45-69). Belo Horizonte: Autentica.

Butler, J. (2002). Cuerpos que importan. Barcelona: Paidós.

Butler, J. (2004). Deshacer el género. Buenos Aires: Paidós

Connell, R. (1997). Escuelas y justicia social. Madrid: Morata.

Edwards, V. (1995) Las formas del conocimiento en el aula. En E. Rockwell (Coord.), La escuela cotidiana (pp. 117-146). Ciudad de México: Fondo de Cultura Económica

Epstein, D. y Johnson, R. (1998). Schooling sexualities. Buckingham: Open University Press. 
Fausto-Sterling, A. (2006). Cuerpos sexuados. La política de género y la construcción de la sexualidad. Madrid: Melusina.

Fainsod, P. y Busca, M. (2016). Educación para la salud y género. Rosario: Homo Sapiens.

Fine, M. (1999). Sexualidad, educación y mujeres adolescentes. En M. Belausteguigoitia y A. Mingo (Eds.), Géneros prófugos. Feminismo y educación (pp. 237-259). Ciudad de México: PUEG - UNAM.

Fraser, N. (1997). Justitia Interrupta. Reflexiones críticas desde la posición postsocialista. Bogotá: Siglo de Hombres.

González del Cerro, C. y Busca, M. (2017). Más allá del sistema reproductor. Aportes para la enseñanza de la biología desde la perspectuva de género. Rosario: Homo Sapiens.

Hooks, B. (1999). Eros, erotismo e o processo pedagógico. En G. Lopes Louro (Comp.), O Corpo educado. Pedagogias da sexualidade (pp. 71-92). Belo Horizonte: Autentica.

Lasa Álvarez, B. (2016) La incorporación de las escritoras al currículo literario en la educación secundaria: Una tarea pendiente. Cuestiones de género: de la igualdad y de la diferencia, 11, 423-442.

Lavigne, L. (2016) Una etnografía educativa sobre sexualidades, género y educación. La educación sexual integral en la ciudad de Buenos Aires como política de gestión de la sexualidad juvenil (Tesis doctoral). Universidad de Buenos Aires.

Lenoir, Y. (2013). Interdisciplinariedad en educación: Una síntesis de sus especificidades y actualización. Interdisciplina, 1(1), 51-86.

López Cao, M. (2002). La educación artística y la equidad de géneros: un asunto pendiente. Arte, Individuo y Sociedad, 1, 145-171

López Navajas, A. (2014). Análisis de la ausencia de las mujeres en los manuales de la ESO: Una genealogía del conocimiento ocultada. Revista de Educación, 363, 282-308.

López Valero, A., Madrid Izquierdo, J. y Encabo Fernández, E. (1998). El discurso del profesorado del área de lengua y literatura ante la transmisión de géneros en la educación secundaria. Revista Fuentes, 1, 47-60.

Louro, G (1999). O corpo educado. Pedagogías da sexualidade. Belo Horizonte: Auténtica.

Louro, G. (2004). Un corpo estranho, ensaios sobre sexualidade e teoria queer. Belo Horizonte: Autêntica.

Maher, F. y Thompson Tetreault, M. (2001). The feminist classroom. Nueva York, NY: Rowman and Littlefield.

Meinardi, E. (2008). Educación para la salud sexual en la formación de profesores. En E. Meinardi y A. Revel (Comp), Género y educación sexual en las escuelas (pp. 23-56). Buenos Aires: De autor.

Morgade, G. y Alonso, G. (2008). Cuerpos sexuados en la escuela. Buenos Aires: Paidós.

Morgade, G. (2011). Toda educación es sexual. Buenos Aires: La Crujía.

Morgade, G. y Fainsod, P. (2015). Convergencias y divergencias de sentido en los talleres de educación sexual integral de la formación docente. Revista del Instituto de Investigaciones en Ciencias de la Educación, 38, 39-62.

Morgade, G. (2016). Educación sexual integral. Rosario: Homo Sapiens. 
Moss-Racusin, C., Dovidio, J., Brescoll, V., Graham, M. y Handelsman, J. (2012). Science faculty's subtle gender biases favor male students. Proceedings of the national academy of sciences, 109(41), 16474-16479.

Péchin, J. (2014). Sobre la hermenéutica genitalista y sus autentificaciones sexo-genéricas: reflexiones pedagógicas para una crítica de sus condiciones institucionales de perpetuidad. Revista Periódicus, 1(2), 1-15.

Péchin, J. (2013). ¿Cómo construye varones la escuela? Etnografía crítica sobre rituales de masculinización en la escena escolar. Revista Iberoamericana de Educación, 62, 181-202.

Perrenoud, P. (2001). La formación de los docentes en el Siglo XXI. Revista de Tecnología Educativa, 14(3), 503-523.

Piussi, A. (1989). Educare nella diferenza. Gruppo pedagogia della differenza sessuales. Turín: Rosenberg \& Sellier.

Scharagrodsky, P. (2006) Tras las huellas de la educación física escolar argentina. Cuerpo, género y pedagogía (1880-1950). Buenos Aires: Prometeo.

Scharagrodsky, P. (2008). Gobernar es ejercitar. Fragmentos para una historia de la educación física en Iberoamérica. Buenos Aires: Prometeo.

Sirvent, M. T. (1993). La investigación participativa aplicada a la renovación curricular. Revista Latinoamericana de innovaciones educativas. Revista del IICE, 1, 11-74.

Tadeu Da Silva, T. (1999). Documentos de identidad. Una introducción a las teorías del currículo. Belo Horizonte: Autêntica Editorial.

Wainerman, C., Di Virgilio, M. y Chami, N. (2008). La escuela y la educación sexual. Buenos Aires: Manantial.

\section{Breve CV de la autora}

\section{Graciela Morgade}

Doctora en Educación (Facultad de Filosofía y Letras - UBA), Máster en Ciencias Sociales y Educacion (FLACSO-Argentina) y Licenciada en Ciencias de la Educación (Facultad de Filosofía y Letras - UBA). Profesora Adjunta Regular de Investigación y Estadística Educacional II y a cargo del Seminario Educación, Género y Sexualidades del Departamento de Ciencias de la Educación. Profesora de posgrado en temas de Investigación Educativa (Maestría en Pedagogías Críticas y Problemáticas Socieducativas- Facultad de Filosofía y letras- UBA y maestría en Docencia Universitaria -UBA) y de temas de género, educación y sexualidades con especial énfasis en la formación de profesores/as y currículum (Diplomado Virtual Educación y Género. Universidad de Antioquia, Colombia y Seminario Educación y Género. Maestría Virtual en Género y Políticas Públicas - PRIGEPP-FLACSO). Investigadora y especialista en Educación Sexual, con enfoque de Género y Derechos Humanos. ORCID ID: OOOOoo02-2801-1911. Email: gmorgade@filo.uba.ar 